\title{
丙酮氰醇为氰源的卤代烃的氰基取代反应研究
}

\author{
郭芳 由 君* 武文菊喻艳超* 井涁刘波
}

(哈尔滨理工大学材料科学与工程学院＼cjkstart绿色化工技术黑龙江省高校重点实验室＼cjkstart哈尔滨 150040)

\begin{abstract}
摘要 氧基取代反应是有机合成中引入氰基官能团的重要方法. 以丙酮氰醇为氰化试剂, 以脂肪族卤代烷 $\mathrm{R}-\mathrm{X}(\mathrm{X}=$ $\mathrm{Cl}, \mathrm{Br}, \mathrm{I})$ 为底物, 通过亲核取代反应合成一系列氰基化合物. 研究结果表明, 在 $50{ }^{\circ} \mathrm{C}$ 四氢呋喃(THF)与 1,3 -二甲基 咪唑啉酮(DMI)(体积比为 $3: 1$ )组成的混合物为反应溶剂, $\mathrm{LiOH} \cdot \mathrm{H}_{2} \mathrm{O}$ 为碱, 丙酮氰醇与相应的卤代烷反应可以得到氰 化产物，产率达到 70\% 99\%. 该反应使用廉价低毒的丙酮氰醇替代剧毒的氰化钠或其他价格昂贵、使用条件苛刻的氰 化试剂, 且不需要贵金属作催化剂, 反应条件温和, 适用范围广泛, 具有较大的应用价值.
\end{abstract}

关键词＼cjkstart卤代烷; 丙酮氧醇; 氧基取代反应; 氨化产物

\section{Study on the Cyanide Substitution Reaction of Acetone Cannolhydrin as Cyanogen Source}

\author{
Guo, Fang You, Jun* Wu, Wenju Yu, Yanchao* Jing, Bin Liu, Bo \\ (Key Laboratory of Green Chemical Engineering and Technology of Heilongjiang Province, College of \\ Materials Science and Engineering, Harbin University of Science and Technology, Harbin, 150040)
}

\begin{abstract}
The cyano substitution reaction is an important method for introducing cyano functional groups in organic synthesis. In this paper, a series of cyano compounds were synthesized by nucleophilic substitution reaction using acetone cyanohydrin as cyanation reagent and aliphatic haloalkanes $\mathrm{R}-\mathrm{X}(\mathrm{X}=\mathrm{Cl}, \mathrm{Br}, \mathrm{I})$ as substrates. The results show that the cyanation product can be obtained by reacting the acetone cyanohydrin with the corresponding halogenated alkanes at $50{ }^{\circ} \mathrm{C}$, and the yield can reach $70 \% \sim 99 \%$. The reaction solvent is the mixture of tetrahydrofuran (THF) and 1,3-dimethylimidazolinone (DMI) (volume ratio of $3: 1$ ), and the base is $\mathrm{LiOH} \cdot \mathrm{H}_{2} \mathrm{O}$. This reaction uses cheap and low toxicity acetone cyanohydrin instead of highly toxic sodium cyanide or other expensive cyanide reagents requiring harsh conditions and it also does not require precious metals as catalysts. The reaction conditions is mild so that it has extensive application and great value of practical application.
\end{abstract}

Keywords haloalkane; acetone cyanohydrin; cyanide substitution; cyanide

腈类化合物在医药、农药、高分子以及新材料等众 多领域中都有着广泛的应用 ${ }^{[1]}$, 因此向分子中引入氧基 的反应一直是有机合成方法学中的研究热点之一 ${ }^{[2]}$. 现 有制备腈类化合物的方法很多, 其中卤代烷与氧化试剂 发生亲核取代反应是应用较多的合成氰基化合物的方 法. 该方法使用的氧化试剂主要有以下几类: (1)反应活 性较高的金属氯化物 $\mathrm{NaCN}$ 或 $\mathrm{KCN}^{[3]}$, 这类物质属于剧 毒品, 其使用受到严格限制; (2) $\mathrm{CuCN} 、 \mathrm{ZnCN} 、 \mathrm{~K}_{4}[\mathrm{Fe}-$ $(\mathrm{CN})_{6}$ 等金属氰化物 ${ }^{[4]}$, 这类物质毒性较 $\mathrm{NaCN}$ 或 $\mathrm{KCN}$ 低, 但反应活性明显下降, 需要加入贵金属 $\mathrm{Pd}$ 等作催化
剂; (3)三甲基氰硅烷等有机氧化物 ${ }^{[5]}$, 这些物质在催化 剂存在下有较好的反应活性, 但昂贵的价格及无水无氧 等严格的操作条件，使其仅适用于实验室或者小规模制 备腈类化合物，严重限制了这类氧化试剂的大规模产业 化应用.

丙酮氰醇 $[6-8]$ 是生产丙烯腈的副产物，可以溶于水 及多种有机溶剂, 并且它沸点较高不易挥发, 毒性较低, 价格低廉, 是一种很有潜力的氰化试剂. 目前丙酮氰醇 与卤代烷发生亲核取代反应的研究不多, 其中 Dowd 等 ${ }^{[7]}$ 使用 $\mathrm{TMG}$ 作碱, $\alpha$-溴代羧酸酯或者活泼的苠基卤代

\footnotetext{
* Corresponding authors. E-mail: youjunjun@126.com; yychao136@163.com

Received February 19, 2021; revised March 23, 2021; published online April 16, 2021.

Project supported by the Natural Science Foundation of Heilongjiang Province (No. LH2019B010), and the National Natural Science Foundation of China (Nos. 21908034, 22008045).

黑龙江省自然科学基金(No. LH2019B010)、国家自然科学基金(Nos. 21908034, 22008045)资助项目.
} 
烷作底物，可以得到 71\% 93\%的氰代产物; Éismont 等 ${ }^{[8]}$ 使用 $\mathrm{K}_{2} \mathrm{CO}_{3}$ 作碱, 18-C-6 等作相转移催化剂, 苯甲 基卤代烷可以高产率地转化为氧代产物, 该方法具有反 应条件温和, 收率较高等优点. 本课题组也试图将上述 两种方法用于非苯甲基的脂肪族伯卤代烷的氰代反应 研究, 但几乎不能得到目标产物. 因此寻找在简单碱作 用下，适用范围更加广泛，反应条件更加温和，以丙酮 氧醇作氰化试剂直接与卤代烷发生氰基取代反应成为 本文研究的核心内容.

\section{1 结果与讨论}

以溴化苄制备苯乙腈的反应为模型反应, 考察了碱 和反应溶剂的种类与用量、丙酮氧醇与底物的投料比以 及温度等因素对反应的影响，由此确定最优反应条件.

丙酮氰醇在碱性条件下可以分解产生氰根离子, 所 以以丙酮氧醇为氧化试剂的反应一般是在碱性条件下 完成. 本文首先对常见的路易斯碱进行了篮选, 找出最 佳碱, 并对最佳碱的用量进行了考察, 实验结果如表 1 所示.

表 1 碱的种类及用量的考察 ${ }^{a}$

Table 1 Investigation of base types and dosages

\begin{tabular}{|c|c|c|c|c|}
\hline & $\mathrm{Br}+(\mathrm{CH}$ & $\begin{array}{c}\text { Base } \\
\mathbf{3} \\
\text { solve }\end{array}$ & & $\mathrm{N}$ \\
\hline Entry & Base & $\begin{array}{l}\text { Amount of } \\
\text { base/equiv. }\end{array}$ & Time/h & Yield/\% \\
\hline 1 & 咪唑 & 1.5 & 24 & Trace \\
\hline 2 & $N$-丁基咪唑 & 1.5 & 24 & Trace \\
\hline 3 & 无水哌嗪 & 1.5 & 10 & 20 \\
\hline 4 & 三乙胺 & 1.5 & 10 & 23 \\
\hline 5 & 吡啶 & 1.5 & 10 & 40 \\
\hline 6 & 叔丁醇钾 & 1.5 & 10 & 25 \\
\hline 7 & DMAP & 1.5 & 10 & 53 \\
\hline 8 & $\mathrm{NaOH}$ & 1.5 & 10 & 62 \\
\hline 9 & $\mathrm{LiOH} \cdot \mathrm{H}_{2} \mathrm{O}$ & 1.1 & 6 & 85 \\
\hline 10 & $\mathrm{LiOH} \cdot \mathrm{H}_{2} \mathrm{O}$ & 1.2 & 5 & 89 \\
\hline 11 & $\mathrm{LiOH} \cdot \mathrm{H}_{2} \mathrm{O}$ & 1.3 & 4 & 92 \\
\hline 12 & $\mathrm{LiOH} \cdot \mathrm{H}_{2} \mathrm{O}$ & 1.5 & 3 & 98 \\
\hline 13 & $\mathrm{LiOH} \cdot \mathrm{H}_{2} \mathrm{O}$ & 1.8 & 3 & 95 \\
\hline 14 & $\mathrm{LiOH}$ & 1.5 & 3 & 98 \\
\hline 15 & $\mathrm{CH}_{3} \mathrm{COONa}$ & 1.5 & 10 & Trace \\
\hline 16 & $\mathrm{Na}_{2} \mathrm{CO}_{3}$ & 1.5 & 10 & Trace \\
\hline 17 & $\mathrm{~K}_{2} \mathrm{CO}_{3}$ & 1.5 & 10 & Trace \\
\hline 18 & $\mathrm{NaHCO}_{3}$ & 1.5 & 10 & Trace \\
\hline $19^{b}$ & $\mathrm{NaOH}$ & 1.5 & 10 & 60 \\
\hline $20^{b}$ & $\mathrm{CH}_{3} \mathrm{COONa}$ & 1.5 & 10 & Trace \\
\hline $21^{b}$ & $\mathrm{Na}_{2} \mathrm{CO}_{3}$ & 1.5 & 10 & Trace \\
\hline
\end{tabular}

${ }^{a}$ Reaction conditions: $1(1 \mathrm{mmol})$ and $2(1.5 \mathrm{mmol})$ in solvent (DMI : THF, $V: V=3: 1,12 \mathrm{~mL}$ ). The temperature was $50{ }^{\circ} \mathrm{C} .{ }^{b}$ Five drops of distilled $\mathrm{H}_{2} \mathrm{O}$ were added.
本工作考察了 13 种常见的路易斯碱对取代反应的 影响(表 1). 研究结果表明, 碱性较弱的咪唑、 $N$-丁基咪 坐几乎不能使反应发生，经过 $24 \mathrm{~h}$ 的反应没有可检测的 氭化产物(Entries 1,2), 碱性稍强的脂肪胺无水哌嗪、三 乙胺也仅有较弱的反应活性，氰化产物收率只有 $20 \%$ 左 右(Entries 3,4). 比较而言, 吡啶类衍生物具有较强的反 应活性，吡啶为碱时氧化产物收率达到 $40 \%$ (Entry 5), 而 4-二甲氨基吡啶(DMAP)为碱时达到 53\% (Entry 7).

当氢氧化钠作碱时反应 $10 \mathrm{~h}$, 氧化产物收率达到 $62 \%$ (Entry 8), 而使用 $\mathrm{LiOH} \cdot \mathrm{H}_{2} \mathrm{O}$ 时产物的收率提高到 $85 \%$ 以上(Entries $9 \sim 13$ ). 随着 $\mathrm{LiOH} \cdot \mathrm{H}_{2} \mathrm{O}$ 的用量由 1.1 equiv. 增加至 1.5 equiv., 氧化产物的收率由 $85 \%$ 提高至 $98 \%$, 反应时间由 $6 \mathrm{~h}$ 缩短到 $3 \mathrm{~h}$; 继续增加 $\mathrm{LiOH} \cdot \mathrm{H}_{2} \mathrm{O}$ 的量, 反应时间没有明显改变而氰化产物收率略有下降 (Entry 13). 其他无机碱 $\mathrm{CH}_{3} \mathrm{COONa} 、 \mathrm{Na}_{2} \mathrm{CO}_{3} 、 \mathrm{~K}_{2} \mathrm{CO}_{3}$ 、 $\mathrm{NaHCO}_{3}$ 对反应没有明显地促进作用(Entries $15 \sim 18$ ). 具有最强碱性的叔丁醇钾作碱时, 氧化产物收率只有 $25 \%$ (Entry 6). 上述实验结果表明，不同的碱对反应收 率有着明显的影响, 反应以 $\mathrm{LiOH} \cdot \mathrm{H}_{2} \mathrm{O}$ 为碱得到最高的 收率.

为了检验 $\mathrm{LiOH} \cdot \mathrm{H}_{2} \mathrm{O}$ 中微量的水是否对反应产生影 响, 检测了无水 $\mathrm{LiOH}$ 的反应活性(Entry 14). 实验结果 表明, 无水 $\mathrm{LiOH}$ 与 $\mathrm{LiOH} \cdot \mathrm{H}_{2} \mathrm{O}$ 的反应活性几乎没有差 别. 进一步考察了在其他无机碱 $\left(\mathrm{NaOH} 、 \mathrm{CH}_{3} \mathrm{COONa}\right.$ 、 $\mathrm{Na}_{2} \mathrm{CO}_{3}$ ) 中加入微量水是否促进反应(Entries 19 21), 研究结果显示少量水对该反应活性没有明显促进作用.

研究发现不同溶剂为反应介质时反应产率差别很 大. 为系统考察溶剂对反应的影响, 以 7 种常用的非质 子性极性溶剂或者其混合物作为反应介质，考察溶剂对 反应收率的影响. 实验采用固定溶剂总量 $(1.0 \mathrm{mmol}$ 反 应物加 $12 \mathrm{~mL}$ 溶剂)的方法, 反应结果如表 2 所示. 结果 表明, 溶剂对反应速度和收率都有重要影响. 使用 1,3二甲基咪唑啉酮(DMI)、 $N$-甲基吡咯烷酮(NMP)和六甲基 磷酸三胺(HMPA)时, 反应以较快的速率高产率地得到目 标产物, 反应时间 $3 \mathrm{~h}$ 时, 产率即可到达 $95 \%$ 以上 (Entries 1 3). 而丙酮、四氢呋喃(THF)和 $\mathrm{CH}_{3} \mathrm{CN}$ 作溶 剂时, 反应几乎不能发生(Entries 5,6)或者收率极低 (Entry 7). 另外, $N, N$-二甲基甲酰胺(DMF)较丙酮、 $\mathrm{THF}$ 和 $\mathrm{CH}_{3} \mathrm{CN}$ 稍好，其反应 $10 \mathrm{~h}$ 后收率可达 $50 \%$ (Entry 4).

为尽量减少高沸点溶剂的用量, 本文以 $\mathrm{DMI}$ 为标 志物研究了其与低沸点溶剂的混合物对反应的影响. 结 果表明, 丙酮和 $\mathrm{CH}_{3} \mathrm{CN}$ 与 DMI 混合时, 反应收率可达 到 64\%和 $67 \%$ (Entries 8,9), 而体积比为 $1: 1$ 的 THF 与 DMI 混合溶剂, 反应速率和产率与纯 DMI 作溶剂时几 乎一致(Entry 10). 继续降低 DMI 用量至 DMI/THF 的体 
表 2 溶剂种类及用量的考察 $a$

Table 2 Investigation of solvent types and dosages

\begin{tabular}{clcl}
\hline Entry & \multicolumn{1}{c}{ Solvent } & Time/h & Yield $\%$ \\
\hline 1 & DMI & 3 & 97 \\
2 & NMP & 3 & 95 \\
3 & HMPA & 3 & 96 \\
4 & DMF & 10 & 50 \\
5 & Acetone & 24 & Trace \\
6 & $\mathrm{CH}_{3} \mathrm{CN}$ & 24 & Trace \\
7 & $\mathrm{THF}$ & 10 & 13 \\
8 & $\mathrm{DMI} /$ Acetone $(V: V=1: 1)$ & 10 & 64 \\
9 & $\mathrm{DMI} / \mathrm{CH}{ }_{3} \mathrm{CN}(V: V=1: 1)$ & 10 & 67 \\
10 & $\mathrm{DMI} / \mathrm{THF}(V: V=1: 1)$ & 3 & 98 \\
11 & $\mathrm{DMI} / \mathrm{THF}(V: V=1: 3)$ & 3 & 98 \\
12 & $\mathrm{DMI} / \mathrm{THF}(V: V=1: 5)$ & 6 & 93 \\
\hline
\end{tabular}

${ }^{a}$ Reaction conditions: $1(1.0 \mathrm{mmol})$ and $2(1.5 \mathrm{mmol})$ in $12 \mathrm{~mL}$ of solvent. $\mathrm{LiOH} \cdot \mathrm{H}_{2} \mathrm{O}$ was $1.5 \mathrm{mmol}$ and temperature was $50{ }^{\circ} \mathrm{C}$.

积比为 $1: 3$, 混合溶剂的性能没有下降(Entry 11), 但当 $\mathrm{DMI} / \mathrm{THF}$ 的体积比达到 $1: 5$ 时, 反应速率明显降低, 产率也有所减少(Entry 12). 因此, 混合溶剂 DMI/THF 最佳体积比为 $1: 3$.

丙酮氰醇的用量和反应温度对氰化反应的也有重 要影响, 实验结果如表 3 所示.

表 3 丙酮氰醇用量和反应温度的考察 $a$

Table 3 Investigation of acetone cyanohydrin dosage and reaction temperature

\begin{tabular}{ccccc}
\hline Entry & 丙酮氧醇/equiv. & Temp. $/{ }^{\circ} \mathrm{C}$ & Time $/ \mathrm{h}$ & Yield $/ \%$ \\
\hline 1 & 1.5 & 25 & 8 & 75 \\
2 & 1.5 & 50 & 3 & 98 \\
3 & 1.5 & 80 & 3 & 94 \\
4 & 1.5 & 120 & 3 & 92 \\
5 & 1 & 50 & 6 & 75 \\
6 & 1.3 & 50 & 5 & 82 \\
7 & 1.8 & 50 & 3.5 & 98 \\
\hline
\end{tabular}

${ }^{a}$ Reaction conditions: $1(1 \mathrm{mmol})$ and $\mathbf{2}$ in $12 \mathrm{~mL}$ of solvent (DMI/THF, $V$ : $V=3: 1) . \mathrm{LiOH} \cdot \mathrm{H}_{2} \mathrm{O}$ was $1.5 \mathrm{mmol}$ and temperature was $50{ }^{\circ} \mathrm{C}$.

在实验过程中发现, 室温时 $\mathrm{LiOH} \cdot \mathrm{H}_{2} \mathrm{O}$ 在反应体系 中溶解度较小, 随着温度的升高, $\mathrm{LiOH} \cdot \mathrm{H}_{2} \mathrm{O}$ 的溶解度 不断提高. 所以, 在反应温度为 $25{ }^{\circ} \mathrm{C}$ 时, 由于 $\mathrm{LiOH} \cdot$ $\mathrm{H}_{2} \mathrm{O}$ 没有完全溶解，反应时间 $8 \mathrm{~h}$ 产率只有 $75 \%$ (Entry 1). 温度升高至 $50{ }^{\circ} \mathrm{C}$ 时, $\mathrm{LiOH} \cdot \mathrm{H}_{2} \mathrm{O}$ 全部溶解, 反应体 系呈透明状态, 产物收率提高至 98\% (Entry 2). 继续升 高温度, 产率有下降趋势(Entries 3,4), 可能与高温下副 反应增加有关.

丙酮氰醇的用量对反应速度及产物收率也有较大 影响. 当反应温度为 $50{ }^{\circ} \mathrm{C}$, 丙酮氰醇按等物质的量加 入时, 反应时间 $6 \mathrm{~h}$ 时, 产率仅为 $75 \%$ (Entry 5). 增加丙 酮氰醇的用量至 $1.3 \mathrm{~mol}$, 反应速率明显加快, 产率也提 高至 $82 \%$ (Entry 6). 当丙酮氧醇的量达到 1.5 equiv.时, 苯乙腈的产率达到 98\% (Entry 2). 继续增加丙酮氰醇的
用量，产率没有明显变化(Entry 7)，但丙酮氧醇的剩余 量增加.

综上所述, 以丙酮氰醇为氰源, 氢氧化锂作碱, 溶 剂为 THF/DMI $(V: V=3: 1)$, 温度为 $50{ }^{\circ} \mathrm{C}$, 反应时间 $3 \mathrm{~h}$, 丙酮氰醇用量为 $1.5 \mathrm{~mol}$ 时, 芐基溴生成苯乙腈的 产率可以达到 $98 \%$.

为考察以卤代烷和丙酮氰醇为氰源的氰化反应的 原料普适性，在上述最优的反应条件下，以不同结构的 卤代烷为反应底物，探索了丙酮氰醇为氧源的氧化反应 的适用范围, 实验结果见表 4. 结果表明, 以丙酮氰醇 为氰源的卤代烷氰化反应的底物适应性很好, 不同结构 的伯卤代烷和活泼的仲卤代烷均能以较高产率得到相 应氧化产物。

总体而言，卤代烷的活性以 $\mathrm{I}, \mathrm{Br}, \mathrm{Cl}$ 的顺序降低, 具体体现在反应时间和产物收率上. 不同氯代烷的反应 时间为 3.5 7.0 h, 氧化产物收率为 71\% 88\% (Entries $1 \sim 12)$. 溴代烷的反应时间缩短为 $3 \sim 6 \mathrm{~h}$, 而氧化产物 收率增加至 75\% 98\%(表 4, Entries 13～26, 表 3, Entry 2). 最活泼的碘代烷反应时间为 $2.5 \sim 5.0 \mathrm{~h}$, 氧化产物收 率为 $80 \% \sim 99 \%$ (Entries 27 36).

亲核取代活性最高的苄基卤代烷具有最高的反应 性，其中反应性最高的苄基碘反应时间为 $2.5 \mathrm{~h}$, 收率达 到 99\% (Entry 27)，活性最差的茮基氯反应时间也只有 $3.5 \mathrm{~h}$, 收率达到 88\% (Entry 1). 三种苄基卤代烷均是本 系列中反应时间最短，氧化产物收率最高的.

由于空间位阻效应，仲卤代烷的氰化反应活性低于 伯卤代烷. 1-苯基溴乙烷、二苯基溴甲烷和 1-苯基碘乙 烷的氰化反应与茮基溴和茮基碘相比反应时间延长, 氰 化产物收率降低(表 4 Entries 14, 15, 29; 表 3 Entry 2).

卤代烷中含有酯基、氰基、卤素等取代基对氰化反 应活性有一定促进作用，这种效应应该是取代基的吸电 子作用使卤代烷的亲核取代活性增加(Entries $3 \sim 6$, 16 20,30). 从研究结果还可以看出, 卤代烷的烷基链 由 4 个碳增长至 18 个碳时, 氯代烷的氰化产物收率由 $71 \%$ 增长为 $77 \%$ (Entries 7 12), 澳代烷由 75\%增长为 $85 \%$ (Entries 21 26), 而碘代烷则由 80\%增长为 $92 \%$ (Entries 31 36).

\section{2 结论}

提供了一条使用低毒的丙酮氰醇作为氰源, 在价格 低廉的 $\mathrm{LiOH} \cdot \mathrm{H}_{2} \mathrm{O}$ 存在下, 通过卤代烷的亲核取代反应 合成系列氰基化合物的技术路线. 该路线反应条件温 和，避免使用剧毒的氰化物，氰化产物产率较高，工艺 简单且原料适用性广，生产成本低，是一条适用于工业 化生产的技术路线. 
表 4 不同卤代烷氰化反应结果 ${ }^{a}$

Table 4 Results of different halogenated cyanide reactions

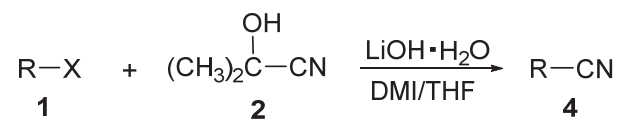

\begin{tabular}{|c|c|c|c|c|}
\hline Entry & Substrate $\mathrm{R}-\mathrm{X}$ & Time/h & Product (4) & Yield/\% \\
\hline 1 & $\mathrm{PhCH}_{2} \mathrm{Cl}$ & 3.5 & $\mathrm{PhCH}_{2} \mathrm{CN}(4 \mathbf{a})$ & 88 \\
\hline 2 & $\mathrm{PhCH}_{2} \mathrm{CH}_{2} \mathrm{Cl}$ & 4.0 & $\mathrm{PhCH}_{2} \mathrm{CH}_{2} \mathrm{CN}(\mathbf{4 b})$ & 85 \\
\hline 3 & $\mathrm{ClCH}_{2} \mathrm{CH}_{2} \mathrm{CN}$ & 4.0 & $\mathrm{CNCH}_{2} \mathrm{CH}_{2} \mathrm{CN}(4 \mathrm{c})$ & 78 \\
\hline 4 & $\mathrm{ClCH}_{2} \mathrm{CH}_{2} \mathrm{Cl}$ & 4.5 & $\mathrm{CNCH}_{2} \mathrm{CH}_{2} \mathrm{CN}(4 \mathrm{c})$ & 77 \\
\hline 5 & $\mathrm{ClCH}_{2} \mathrm{CH}_{2} \mathrm{CH}_{2} \mathrm{CH}_{2} \mathrm{Cl}$ & 3.5 & $\mathrm{CNCH}_{2} \mathrm{CH}_{2} \mathrm{CH}_{2} \mathrm{CH}_{2} \mathrm{CN}(\mathbf{4 d})$ & 79 \\
\hline 6 & $\mathrm{ClCH}_{2} \mathrm{CH}_{2} \mathrm{COOCH}_{2} \mathrm{CH}_{3}$ & 4.0 & $\mathrm{CNCH}_{2} \mathrm{CH}_{2} \mathrm{COOCH}_{2} \mathrm{CH}_{3}(\mathbf{4 e})$ & 77 \\
\hline 7 & $\mathrm{ClCH}_{2} \mathrm{CH}_{2} \mathrm{CH}_{2} \mathrm{CH}_{3}$ & 7.0 & $\mathrm{CNCH}_{2} \mathrm{CH}_{2} \mathrm{CH}_{2} \mathrm{CH}_{3}(\mathbf{4 f})$ & 71 \\
\hline 8 & $\mathrm{Cl}\left(\mathrm{CH}_{2}\right)_{7} \mathrm{CH}_{3}$ & 6.5 & $\mathrm{CN}\left(\mathrm{CH}_{2}\right)_{7} \mathrm{CH}_{3}(\mathbf{4 g})$ & 72 \\
\hline 9 & $\mathrm{Cl}\left(\mathrm{CH}_{2}\right)_{11} \mathrm{CH}_{3}$ & 5.5 & $\mathrm{CN}\left(\mathrm{CH}_{2}\right)_{11} \mathrm{CH}_{3}(\mathbf{4 h})$ & 72 \\
\hline 10 & $\mathrm{Cl}\left(\mathrm{CH}_{2}\right)_{13} \mathrm{CH}_{3}$ & 5.0 & $\mathrm{CN}(\mathrm{CH} 2)_{13} \mathrm{CH}_{3}(4 \mathbf{i})$ & 74 \\
\hline 11 & $\mathrm{Cl}\left(\mathrm{CH}_{2}\right)_{15} \mathrm{CH}_{3}$ & 4.5 & $\mathrm{CN}\left(\mathrm{CH}_{2}\right)_{15} \mathrm{CH}_{3}(\mathbf{4 j})$ & 76 \\
\hline 12 & $\mathrm{Cl}\left(\mathrm{CH}_{2}\right)_{17} \mathrm{CH}_{3}$ & 4.0 & $\mathrm{CN}\left(\mathrm{CH}_{2}\right)_{17} \mathrm{CH}_{3}(\mathbf{4 k})$ & 77 \\
\hline 13 & $\mathrm{PhCH}_{2} \mathrm{CH}_{2} \mathrm{CH}_{2} \mathrm{Br}$ & 3.0 & $\mathrm{PhCH}_{2} \mathrm{CH}_{2} \mathrm{CH}_{2} \mathrm{CN}$ (4l) & 91 \\
\hline 14 & $\mathrm{PhCHBrCH}_{3}$ & 4.0 & $\mathrm{PhCHCNCH}_{3}(4 \mathrm{~m})$ & 91 \\
\hline 15 & $\mathrm{PhCHBrPh}$ & 5.0 & $\mathrm{PhCHCNPh}(\mathbf{4 n})$ & 93 \\
\hline 16 & $\mathrm{BrCH}_{2} \mathrm{CH}_{2} \mathrm{COOCH}_{3}$ & 4.0 & $\mathrm{CNCH}_{2} \mathrm{CH}_{2} \mathrm{COOCH}_{3}(\mathbf{4 o})$ & 85 \\
\hline 17 & $\mathrm{BrCH}_{2} \mathrm{CH}_{2} \mathrm{CH}_{2} \mathrm{COOCH}_{3}$ & 3.5 & $\mathrm{CNCH}_{2} \mathrm{CH}_{2} \mathrm{CH}_{2} \mathrm{COOCH}_{3}(\mathbf{4} \mathbf{p})$ & 89 \\
\hline 18 & $\mathrm{Br}\left(\mathrm{CH}_{2}\right)_{6} \mathrm{COOCH}_{2} \mathrm{CH}_{3}$ & 3.0 & $\left.\mathrm{CN}\left(\mathrm{CH}_{2}\right)_{6} \mathrm{COOCH}_{2} \mathrm{CH}_{3} \mathbf{( 4 q}\right)$ & 93 \\
\hline 19 & $\mathrm{BrCH}_{2} \mathrm{CH}_{2} \mathrm{Br}$ & 4.0 & $\mathrm{CNCH}_{2} \mathrm{CH}_{2} \mathrm{CN}(4 \mathbf{c})$ & 80 \\
\hline 20 & $\mathrm{BrCH}_{2} \mathrm{CH}_{2} \mathrm{CH}_{2} \mathrm{CH}_{2} \mathrm{Br}$ & 3.0 & $\mathrm{CNCH}_{2} \mathrm{CH}_{2} \mathrm{CH}_{2} \mathrm{CH}_{2} \mathrm{CN}(\mathbf{4 d})$ & 85 \\
\hline 21 & $\mathrm{BrCH}_{2} \mathrm{CH}_{2} \mathrm{CH}_{2} \mathrm{CH}_{3}$ & 6.0 & $\mathrm{CNCH}_{2} \mathrm{CH}_{2} \mathrm{CH}_{2} \mathrm{CH}_{3}(\mathbf{4 f})$ & 75 \\
\hline 22 & $\mathrm{Br}\left(\mathrm{CH}_{2}\right)_{7} \mathrm{CH}_{3}$ & 5.5 & $\mathrm{CN}\left(\mathrm{CH}_{2}\right)_{7} \mathrm{CH}_{3}(\mathbf{4 g})$ & 77 \\
\hline 23 & $\mathrm{Br}\left(\mathrm{CH}_{2}\right)_{11} \mathrm{CH}_{3}$ & 4.5 & $\mathrm{CN}\left(\mathrm{CH}_{2}\right)_{11} \mathrm{CH}_{3}(\mathbf{4 h})$ & 79 \\
\hline 24 & $\mathrm{Br}\left(\mathrm{CH}_{2}\right)_{13} \mathrm{CH}_{3}$ & 4.0 & $\mathrm{CN}\left(\mathrm{CH}_{2}\right)_{13} \mathrm{CH}_{3}(\mathbf{4 i})$ & 81 \\
\hline 25 & $\mathrm{Br}\left(\mathrm{CH}_{2}\right)_{15} \mathrm{CH}_{3}$ & 3.5 & $\mathrm{CN}\left(\mathrm{CH}_{2}\right)_{15} \mathrm{CH}_{3}(\mathbf{4} \mathbf{j})$ & 83 \\
\hline 26 & $\mathrm{Br}\left(\mathrm{CH}_{2}\right)_{17} \mathrm{CH}_{3}$ & 3.0 & $\mathrm{CN}\left(\mathrm{CH}_{2}\right)_{17} \mathrm{CH}_{3}(\mathbf{4 k})$ & 86 \\
\hline 27 & $\mathrm{PhCH}_{2} \mathrm{I}$ & 2.5 & $\mathrm{PhCH}_{2} \mathrm{CN}(\mathbf{4 a})$ & 99 \\
\hline 28 & $\mathrm{PhCH}_{2} \mathrm{CH}_{2} \mathrm{CH}_{2} \mathrm{I}$ & 2.5 & $\mathrm{PhCH}_{2} \mathrm{CH}_{2} \mathrm{CH}_{2} \mathrm{CN}$ (4I) & 92 \\
\hline 29 & $\mathrm{PhCHICH}_{3}$ & 3.0 & $\mathrm{PhCHCNCH}_{3}(\mathbf{4 m})$ & 93 \\
\hline 30 & $\mathrm{ICH}_{2} \mathrm{CH}_{2} \mathrm{CH}_{2} \mathrm{COOCH}_{3}$ & 3.0 & $\mathrm{CNCH}_{2} \mathrm{CH}_{2} \mathrm{CH}_{2} \mathrm{COOCH}_{3}(\mathbf{4} \mathbf{p})$ & 90 \\
\hline 31 & $\mathrm{ICH}_{2} \mathrm{CH}_{2} \mathrm{CH}_{2} \mathrm{CH}_{3}$ & 5.0 & $\mathrm{CNCH}_{2} \mathrm{CH}_{2} \mathrm{CH}_{2} \mathrm{CH}_{3}(\mathbf{4 f})$ & 80 \\
\hline 32 & $\mathrm{I}\left(\mathrm{CH}_{2}\right)_{7} \mathrm{CH}_{3}$ & 4.5 & $\mathrm{CN}\left(\mathrm{CH}_{2}\right)_{7} \mathrm{CH}_{3}(\mathbf{4 g})$ & 83 \\
\hline 33 & $\mathrm{I}\left(\mathrm{CH}_{2}\right)_{11} \mathrm{CH}_{3}$ & 4.0 & $\mathrm{CN}\left(\mathrm{CH}_{2}\right)_{11} \mathrm{CH}_{3}(\mathbf{4 h})$ & 85 \\
\hline 34 & $\mathrm{I}\left(\mathrm{CH}_{2}\right)_{13} \mathrm{CH}_{3}$ & 3.5 & $\mathrm{CN}\left(\mathrm{CH}_{2}\right)_{13} \mathrm{CH}_{3}(\mathbf{4 i})$ & 87 \\
\hline 35 & $\mathrm{I}\left(\mathrm{CH}_{2}\right)_{15} \mathrm{CH}_{3}$ & 3.0 & $\mathrm{CN}\left(\mathrm{CH}_{2}\right)_{15} \mathrm{CH}_{3}(\mathbf{4 j})$ & 89 \\
\hline 36 & $\mathrm{I}\left(\mathrm{CH}_{2}\right)_{17} \mathrm{CH}_{3}$ & 2.5 & $\mathrm{CN}\left(\mathrm{CH}_{2}\right)_{17} \mathrm{CH}_{3}(\mathbf{4 k})$ & 92 \\
\hline
\end{tabular}

${ }^{a}$ Reaction conditions: $1(1.0 \mathrm{mmol})$ and $2(1.5 \mathrm{mmol})$ in $12 \mathrm{~mL}$ of DMI/THF $(V: \quad V=3: 1), \mathrm{LiOH} \cdot \mathrm{H}_{2} \mathrm{O}$ was $1.5 \mathrm{mmol}$ and temperature was $50{ }^{\circ} \mathrm{C}$.

经条件优化得到的卤代烷氧化反应的最佳条件为: 卤代烷、丙酮氰醇和 $\mathrm{LiOH} \cdot \mathrm{H}_{2} \mathrm{O}$ 的投料摩尔比 1.5 : $1.5: 1.0$, 反应溶剂为 THF 和 DMI 的混合溶剂(体积比 为 $3: 1$ ), 反应温度 $50{ }^{\circ} \mathrm{C}$. 在此条件下, 不同的卤代烷 均可得到良好收率的氰化产物。

\section{3 实验部分}

\section{1 仪器与试剂}

${ }^{1} \mathrm{H}$ NMR 和 ${ }^{13} \mathrm{C}$ NMR 用 Bruker AVANCE-300 MHz
核磁共振谱仪测定, 溶剂为 $\mathrm{CDCl}_{3}$; 质谱用 Waters $\mathrm{Mi}-$ cromass GCT Primier 质谱仪检测; 红外光谱用 Avatar370 FT-IR 型红外光谱仪测得. 所用药品和试剂均 为市售的分析纯.

\section{2 实验方法}

称取丙酮氰醇 $0.37 \mathrm{~g}(1.5 \mathrm{mmol})$ 置于 $50 \mathrm{~mL}$ 三口瓶 中, 加入 DMI 与 THF $(V: V=1: 3)$ 混合溶液 $12 \mathrm{~mL}$, 按 比例加入 $1.5 \mathrm{mmol} \mathrm{LiOH} \cdot \mathrm{H}_{2} \mathrm{O}$, 室温摚拌 $1 \mathrm{~h}$ 后加入卤 代烷 1 36 (1.0 mmol), 加热至 $50{ }^{\circ} \mathrm{C}$, 薄层色谱分析 
(TLC)监控反应，反应结束后加入去离子水洗涤，用乙 酸乙酯萃取 $(5 \mathrm{~mL} \times 3)$, 乙酸乙酯层再分别用去离子水 和饱和食盐水洗涤, 有机层用无水 $\mathrm{Na}_{2} \mathrm{SO}_{4}$ 干燥, 过滤, 旋蒸除去溶剂, 经硅胶柱层析分离 $[V$ (石油醚) $: V($ 乙酸 乙酯) $=10 ： 1]$ 得到淡黄色油状液体.

\section{3 化合物的谱图数据}

苯乙腈 $(\mathbf{4 a})^{[9 \mathrm{a}]}$ : 以 $\mathbf{1}, 27$ 为底物时, 分别得 103,116 $\mathrm{mg}$, 产率 $88 \%, 99 \%$. 淡黄色油状液体. ${ }^{1} \mathrm{H} \mathrm{NMR}\left(\mathrm{CDCl}_{3}\right.$, $300 \mathrm{MHz}) \delta: 3.75$ (s, 2H), $7.32 \sim 7.44(\mathrm{~m}, 5 \mathrm{H}) ;{ }^{13} \mathrm{C} \mathrm{NMR}$ $\left(\mathrm{CDCl}_{3}, 75 \mathrm{MHz}\right) \delta: 23.3,117.8,127.7,127.8,128.9$, 129.9; IR (KBr) v: 3091, 3066, 3034, 2922, 2253, 1603, 1497, 1454, 1416, 1076, 1030, 941, 735, 696, 615, 465 $\mathrm{cm}^{-1}$.

3-苯基丙腈(4b) ${ }^{[9 b]}: 111 \mathrm{mg}$, 产率 $85 \%$. 无色油状液 体. ${ }^{1} \mathrm{H}$ NMR $\left(\mathrm{CDCl}_{3}, 300 \mathrm{MHz}\right) \delta: 2.60(\mathrm{t}, J=7.40 \mathrm{~Hz}$, $2 \mathrm{H}), 2.94(\mathrm{t}, J=7.36 \mathrm{~Hz}, 2 \mathrm{H}), 7.21 \sim 7.36(\mathrm{~m}, 5 \mathrm{H}) ;{ }^{13} \mathrm{C}$ NMR (75 MHz, $\left.\mathrm{CDCl}_{3}\right) \delta: 19.3,31.5,119.1,127.2,128.2$, 128.9, 138.1; IR (KBr) v: 3088, 3065, 3030, 2931, 2866, 2247, 1957, 1875, 1813, 1736, 1605, 1497, 1456, 1427, $1080,749,698,590,478 \mathrm{~cm}^{-1}$.

丁二腈 $(4 \mathrm{c})^{[9 \mathrm{c}]}$ : 分别以 $3,4,19$ 为底物得 $63,61,64$ $\mathrm{mg}$, 产率 78\%, 77\%, 80\%. 无色油状液体. ${ }^{1} \mathrm{H}$ NMR $\left(\mathrm{CDCl}_{3}, 300 \mathrm{MHz}\right) \delta: 2.76(\mathrm{~s}, 4 \mathrm{H}) ;{ }^{13} \mathrm{C} \mathrm{NMR}\left(\mathrm{CDCl}_{3}, 75\right.$ MHz) $\delta$ : 14.7, 116.2; IR (KBr) v: 2989, 2951, 2254, 1425, 1005, 1338, 964, 820, 762, 606, $482 \mathrm{~cm}^{-1}$.

己二腈 $(\mathbf{4 d})^{[\mathrm{gd}]}$ : 以 $\mathbf{5 , 2 0}$ 为底物得 $86,92 \mathrm{mg}$, 产率 $79 \%, 85 \%$. 无色油状液体. ${ }^{1} \mathrm{H}$ NMR $\left(\mathrm{CDCl}_{3}, 300 \mathrm{MHz}\right)$ $\delta: 1.81(\mathrm{~s}, 4 \mathrm{H}), 2.42(\mathrm{~s}, 4 \mathrm{H}) ;{ }^{13} \mathrm{C} \mathrm{NMR}\left(\mathrm{CDCl}_{3}, 75 \mathrm{MHz}\right) \delta$ : 16.6, 24.2, 118.7; IR (KBr) v: 2949, 2877, 2247, 1697, $1508,1460,1425,1333,1290,901,897,764,581 \mathrm{~cm}^{-1}$.

3-氧基丙酸乙酯 $(4 \mathrm{e})^{[9 \mathrm{e}]}$ : $98 \mathrm{mg}$, 产率 77\%. 淡黄色 油状液体 4e. ${ }^{1} \mathrm{H}$ NMR $\left(\mathrm{CDCl}_{3}, 300 \mathrm{MHz}\right) \delta: 1.27$ (t, $J=$ $7.13 \mathrm{~Hz}, 3 \mathrm{H}$ ), 2.65 (s, 4H), 4.19 (q, $J=7.09 \mathrm{~Hz}, 2 \mathrm{H}$ ); ${ }^{13} \mathrm{C}$ NMR $\left(\mathrm{CDCl}_{3}, 75 \mathrm{MHz}\right) \delta$ : 13.0, 14.1, 30.2, 61.5, 118.5, 170.0; IR (KBr) v: 2985, 2926, 2854, 2251, 1736, 1686, $1421,1377,1252,1200,854,596 \mathrm{~cm}^{-1}$.

戊腈 $(\mathbf{4 f})^{[9 f]}$ : 以 $7,21,31$ 为底物得 $59,62,67 \mathrm{mg}$, 产 率 71\%,75\%,80\%。淡黄色油状液体 4f. ${ }^{1} \mathrm{H}$ NMR $\left(\mathrm{CDCl}_{3}, 300 \mathrm{MHz}\right) \delta: 0.93(\mathrm{t}, J=7.20 \mathrm{~Hz}, 3 \mathrm{H}), 1.40 \sim 1.52$ (m, $2 \mathrm{H}), 1.7 \sim 1.86(\mathrm{~m}, 2 \mathrm{H}), 3.41(\mathrm{t}, J=7.02 \mathrm{~Hz}, 2 \mathrm{H}) ;{ }^{13} \mathrm{C}$ $\mathrm{NMR}\left(\mathrm{CDCl}_{3}, 75 \mathrm{MHz}\right) \delta: 13.2,16.8,21.8,27.4,119.8$; IR (KBr) v: 2993, 2941, 2243, 1460, 1383, 1192, 1142, 976, $878,696,560 \mathrm{~cm}^{-1}$.

壬腈 $(\mathbf{4 g})^{[\mathrm{gg}]}$ : 以 $\mathbf{8 , 2 2}, 32$ 为底物得 $100,107,116 \mathrm{mg}$, 产率 72\%, 77\%, 83\%. 淡黄色油状液体 $4 \mathrm{gg} .{ }^{1} \mathrm{H}$ NMR
$\left(\mathrm{CDCl}_{3}, 300 \mathrm{MHz}\right) \delta: 0.89(\mathrm{t}, J=6.62 \mathrm{~Hz}, 3 \mathrm{H}), 1.29(\mathrm{~s}$, $8 \mathrm{H}), 1.44$ (br, s, $2 \mathrm{H}), 1.63 \sim 1.67(\mathrm{~m}, 2 \mathrm{H}), 2.32(\mathrm{t}, J=7.05$ $\mathrm{Hz}, 2 \mathrm{H}) ;{ }^{13} \mathrm{C} \mathrm{NMR}\left(\mathrm{CDCl}_{3}, 75 \mathrm{MHz}\right) \delta: 14.1,17.1,22.7$, 25.4, 28.7, 29.0, 31.7, 119.9; IR (KBr) v: 2955, 2928, 2856, 2247, 1466, 1427, 1379, 1121, $723 \mathrm{~cm}^{-1}$.

十二烷腈 $(\mathbf{4 h})^{[9 \mathrm{~h}]}$ : 以 $\mathbf{9}, \mathbf{2 3}, \mathbf{3 3}$ 为底物得 141,155 , $166 \mathrm{mg}$, 产率 72\%, 79\%, 85\%. 无色油状液体. ${ }^{1} \mathrm{H}$ NMR $\left(\mathrm{CDCl}_{3}, 300 \mathrm{MHz}\right) \delta: 0.87(\mathrm{t}, J=6.08 \mathrm{~Hz}, 3 \mathrm{H}), 1.25$ (s, $16 \mathrm{H}), 1.43(\mathrm{br}, \mathrm{s}, 2 \mathrm{H}), 1.60 \sim 1.69(\mathrm{~m}, 2 \mathrm{H}), 2.32(\mathrm{t}, J=$ $7.04 \mathrm{~Hz}, 2 \mathrm{H}) ;{ }^{13} \mathrm{C} \mathrm{NMR}\left(\mathrm{CDCl}_{3}, 75 \mathrm{MHz}\right) \delta: 14.1,17.2$, 22.7, 25.4, 28.7, 28.8, 29.3, 29.5, 29.6, 31.9, 119.8; IR (KBr) $v$ : 2926, 2854, 2247, 1466, 1427, 1377, 1122, 721 $\mathrm{cm}^{-1}$.

十五烷腈 $(\mathbf{4 i})^{[9 i]}$ : 以 $\mathbf{1 0}, \mathbf{2 4}, \mathbf{3 4}$ 为底物得 165,181 , $194 \mathrm{mg}$, 产率 74\%, 81\%，87\%. 白色固体，m.p. 22.4 $23.2{ }^{\circ} \mathrm{C}$ (lit. $\left.\left.{ }^{\left[{ }^{j}\right]}\right] 20 \sim 24{ }^{\circ} \mathrm{C}\right) ;{ }^{1} \mathrm{H}$ NMR $\left(\mathrm{CDCl}_{3}, 300 \mathrm{MHz}\right)$ $\delta: 0.88(\mathrm{t}, J=6.17 \mathrm{~Hz}, 3 \mathrm{H}), 1.26(\mathrm{~s}, 20 \mathrm{H}), 1.44$ (br, s, 2H), $1.60 \sim 1.68(\mathrm{~m}, 2 \mathrm{H}), 2.33(\mathrm{t}, J=7.09 \mathrm{~Hz}, 2 \mathrm{H}) ;{ }^{13} \mathrm{C}$ NMR $\left(\mathrm{CDCl}_{3}, 75 \mathrm{MHz}\right) \delta: 14.1,17.1,22.7,25.4,28.7,28.8$, 29.3, 29.4, 29.5, 29.6, 29.7, 31.9, 119.8; IR (KBr) v: 2926, $2856,2247,1466,1431,1377,721 \mathrm{~cm}^{-1}$.

十七烷腈 $(\mathbf{4 j})^{[9 k]}$ : 以 $\mathbf{1 1}, \mathbf{2 5}, \mathbf{3 5}$ 为底物得 191,209 , $225 \mathrm{mg}$, 产率 76\%, 83\%, 89\%. 白色固体, m.p. 33.4 $34.1{ }^{\circ} \mathrm{C}$ (lit. $\left.{ }^{[9]} 32 \sim 33{ }^{\circ} \mathrm{C}\right) ;{ }^{1} \mathrm{H} \mathrm{NMR}\left(\mathrm{CDCl}_{3}, 300 \mathrm{MHz}\right)$ $\delta: 0.88(\mathrm{t}, J=6.15 \mathrm{~Hz}, 3 \mathrm{H}), 1.26(\mathrm{~s}, 24 \mathrm{H}), 1.44(\mathrm{br}, \mathrm{s}, 2 \mathrm{H})$, $1.60 \sim 1.68(\mathrm{~m}, 2 \mathrm{H}), 2.33(\mathrm{t}, J=7.05 \mathrm{~Hz}, 2 \mathrm{H}) ;{ }^{13} \mathrm{C}$ NMR $\left(\mathrm{CDCl}_{3}, 75 \mathrm{MHz}\right) \delta: 14.1,17.2,22.7,25.4,28.7,28.8$, 29.3, 29.4, 29.5, 29.6, 29.7, 32.0, 119.9; IR (KBr) v: 2916, $2848,2245,1471,1419,1379,1329,717 \mathrm{~cm}^{-1}$.

十九烷腈 $(\mathbf{4 k})^{[9 \mathrm{i}]}$ ：以 12, 26, 36 为底物得 216,240 , $257 \mathrm{mg}$, 产率 77\%, 86\%, 92\%. 白色固体, m.p. 41.7 $42.8{ }^{\circ} \mathrm{C}$ (lit. $\left.{ }^{[\mathrm{m}]} 41.6 \sim 44.3{ }^{\circ} \mathrm{C}\right) ;{ }^{1} \mathrm{H}$ NMR $\left(\mathrm{CDCl}_{3}, 300\right.$ MHz) $\delta: 0.88(\mathrm{t}, J=5.54 \mathrm{~Hz}, 3 \mathrm{H}), 1.26(\mathrm{~s}, 28 \mathrm{H}), 1.44(\mathrm{br}$, s, $2 \mathrm{H}), 1.61 \sim 1.70(\mathrm{~m}, 2 \mathrm{H}), 2.33(\mathrm{t}, J=7.03 \mathrm{~Hz}, 2 \mathrm{H}) ;{ }^{13} \mathrm{C}$ NMR $\left(\mathrm{CDCl}_{3}, 75 \mathrm{MHz}\right) \delta: 14.2,17.2,22.7,25.4,28.7$, 28.8, 29.4, 29.6, 29.7, 32.0, 119.9; IR (KBr) v: 2918, 2850, $2247,1468,1377,1267,721 \mathrm{~cm}^{-1}$.

4-苯基丁腈 $(\mathbf{4 I})^{[9 b]}$ : 以 $\mathbf{1 3}, \mathbf{2 8}$ 为底物得 $132,134 \mathrm{mg}$, 产率 $91 \%, 92 \%$. 淡黄色油状液体. ${ }^{1} \mathrm{H}$ NMR $\left(\mathrm{CDCl}_{3}, 300\right.$ MHz) $\delta: 1.93 \sim 2.04(\mathrm{~m}, 2 \mathrm{H}), 2.31(\mathrm{t}, J=7.09 \mathrm{~Hz}, 2 \mathrm{H})$, 2.78 (t, $J=7.40 \mathrm{~Hz}, 2 \mathrm{H}), 7.17 \sim 7.34(\mathrm{~m}, 5 \mathrm{H}) ;{ }^{13} \mathrm{C}$ NMR $\left(\mathrm{CDCl}_{3}, 75 \mathrm{MHz}\right) \delta: 16.3,26.9,33.9,119.5,126.5,128.4$, 128.6, 139.7; IR (KBr) v: 3063, 3028, 2929, 2868, 2247, $1603,1497,1454,1425,1248,1082,912,750,700,490$ $\mathrm{cm}^{-1}$. 
$\alpha$-甲基苯腈 $(\mathbf{4 m})^{[9 \mathrm{a}]}$ ：以 14, 29 为底物得 $119,122 \mathrm{mg}$, 产率 91\%, 93\%. 无色油状液体. ${ }^{1} \mathrm{H}$ NMR $\left(\mathrm{CDCl}_{3}, 300\right.$ MHz) $\delta: 1.65(\mathrm{~d}, J=7.32 \mathrm{~Hz}, 3 \mathrm{H}), 3.91(\mathrm{q}, \quad J=7.24 \mathrm{~Hz}$, $1 \mathrm{H}), 7.33 \sim 7.42(\mathrm{~m}, 5 \mathrm{H}) ;{ }^{13} \mathrm{C} \mathrm{NMR}\left(\mathrm{CDCl}_{3}, 75 \mathrm{MHz}\right) \delta$ : 21.5, 31.3, 121.6, 126.7, 128.1, 129.2, 137.1; IR (KBr) $v$ : 3091, 3034, 2987, 2939, 2877, 2241, 1959, 1880, 1811, $1672,1585,1385,1279,1182,1130,752,623,571 \mathrm{~cm}^{-1}$.

二苯乙腈 $(4 \mathrm{n})^{[9 \mathrm{f}]}$ : $180 \mathrm{mg}$, 产率 $93 \%$. 白色固体 $4 \mathrm{n}$, m.p. $67.8 \sim 69.3$ (lit. $\left.{ }^{[\mathrm{n}]} 67 \sim 69{ }^{\circ} \mathrm{C}\right) ;{ }^{1} \mathrm{H} \mathrm{NMR}\left(\mathrm{CDCl}_{3}, 300\right.$ $\mathrm{MHz}) \delta: 5.15(\mathrm{~s}, 1 \mathrm{H}), 7.31 \sim 7.41(\mathrm{~m}, 10 \mathrm{H}) ;{ }^{13} \mathrm{C} \mathrm{NMR}$ $\left(\mathrm{CDCl}_{3}, 75 \mathrm{MHz}\right) \delta: 42.6,119.7,127.8,128.3,129.2$, 135.9; IR(KBr) v: 3076, 3028, 2933, 2243, 1952, 1873, 1805, 1751, 1659, 1597, 1493, 1454, 1178, 1078, 744, 698, $540 \mathrm{~cm}^{-1}$.

3-氭基丙酸甲酯(4o $)^{[90]}$ : $96 \mathrm{mg}$, 产率 $85 \%$. 淡黄色 油状液体. ${ }^{1} \mathrm{H} \mathrm{NMR}\left(\mathrm{CDCl}_{3}, 300 \mathrm{MHz}\right) \delta: 2.62 \sim 2.70(\mathrm{~m}$, $4 \mathrm{H}), 3.73(\mathrm{~s}, 3 \mathrm{H}) ;{ }^{13} \mathrm{C} \mathrm{NMR}\left(\mathrm{CDCl}_{3}, 75 \mathrm{MHz}\right) \delta: 13.0$, 29.8, 52.3, 118.4, 170.5; IR (KBr) v: 3117, 3063, 2962, 2254, 1664, 1578, 1508, 1473, 1387, 1279, 1180, 1132, $1053,883,746,683 \mathrm{~cm}^{-1}$.

4-氭基丁酸甲酯 $(\mathbf{4 p})^{[9 \mathrm{p}]}$ : 以 $\mathbf{1 7 , 3 0}$ 为底物得 113,114 $\mathrm{mg}$, 产率 $89 \%, 90 \%$. 淡黄色油状液体. ${ }^{1} \mathrm{H} \mathrm{NMR}\left(\mathrm{CDCl}_{3}\right.$, $300 \mathrm{MHz}) \delta$ : $1.96(\mathrm{t}, J=5.72 \mathrm{~Hz}, 2 \mathrm{H}), 2.42 \sim 2.50(\mathrm{~m}$, $4 \mathrm{H}), 3.67(\mathrm{~s}, 3 \mathrm{H}) ;{ }^{13} \mathrm{C} \mathrm{NMR}\left(\mathrm{CDCl}_{3}, 75 \mathrm{MHz}\right) \delta: 16.7$, 20.7, 32.1, 51.4, 118.9, 172.4; IR (KBr) v: 2956, 2850, 2247, 1736, 1439, 1375, 1321, 1167, 1061, 868, 781, 590 $\mathrm{cm}^{-1} ; \mathrm{MS} m / z: 149.95[\mathrm{M}+\mathrm{Na}]^{+}$.

7-氰基庚酸乙酯(4q) ${ }^{[9 q]}: 170 \mathrm{mg}$, 产率 $93 \%$. 淡黄色 油状液体. ${ }^{1} \mathrm{H}$ NMR $\left(\mathrm{CDCl}_{3}, 300 \mathrm{MHz}\right) \delta: 1.25(\mathrm{t}, J=7.04$ $\mathrm{Hz}, 3 \mathrm{H}), 1.34 \sim 1.38(\mathrm{~m}, 2 \mathrm{H}), 1.42 \sim 1.49(\mathrm{~m}, 2 \mathrm{H}), 1.59 \sim$ $1.69(\mathrm{~m}, 4 \mathrm{H}), 2.27 \sim 2.36(\mathrm{~m}, 4 \mathrm{H}), 4.12(\mathrm{q}, J=7.02$ $\mathrm{Hz}, 2 \mathrm{H}) ;{ }^{13} \mathrm{C} \mathrm{NMR}\left(\mathrm{CDCl}_{3}, 75 \mathrm{MHz}\right) \delta: 14.1,16.9,24.4$, 25.0, 28.1, 28.2, 34.0, 60.1, 119.6, 173.4; IR (KBr) $v$ : 2937, 2866, 2247, 1732, 1464, 1373, 1254, 1188, 1097, 1036, 858, $729 \mathrm{~cm}^{-1}$; MS m/z: $206.10[\mathrm{M}+\mathrm{Na}]^{+}$.

辅助材料(Supporting Information) 所有产物的 ${ }^{1} \mathrm{H}$ $\mathrm{NMR},{ }^{13} \mathrm{C} \mathrm{NMR}$, IR 以及 MS 谱图. 这些材料可以免费从 本刊网站(http://sioc-journal.cn/)上下载.

\section{References}

[1] (a) Fleming, F. F.; Yao, L.; Ravikumar, P. C. J. Med. Chem. 2010, 53,7902 .

(b) Dhillon, S.; Weber, J. Drugs 2009, 69, 2103.

(c) Ma, D. Y. Chin. J. Org. Chem. 2008, 28, 1439 (in Chinese). (马大友, 有机化学, 2008, 28, 1439).

[2] (a) Yu, Z. W.; Li, L. Y.; Shen, Z. M. Chin. J. Org. Chem. 2017, 37,
1273 (in Chinese).

(俞峥炜, 李林奕, 沈增明, 有机化学, 2017, 37, 1273.)

(b) Yan, G.; Zhang, Y.; Wang, J. Adv. Synth. Catal. 2017, 359, 4068. (c) Zhou, H. Y.; Li, N. N.; Yang, J. Y. Chin. J. Org. Chem. 2016, 36, 502 (in Chinese).

(周红艳, 李娜娜, 杨靖亚, 有机化学, 2016, 36, 502.)

(d) Wang, H. S.; Zeng, J. E. Chin. J. Org. Chem. 2012, 32 ,934 (in Chinese).

(王宏社，曾君娥，有机化学, 2012, 32, 934.)

(e) Fang, G. N.; You, J.; Yu, Y. C.; Jing, J. K.; Liu, B.; Wu, W. J. Chin. J. Org. Chem. 2020, 40, 2871 (in Chinese).

(房观念, 由君, 喻艳超, 荆军凯, 刘波, 武文菊, 有机化学, 2020, 40, 2871.)

[3] (a) Shimizu, S.; Kito, K.; Sasaki, Y. Chem. Commun. 1997, 17, 1629.

(b) Fort, Y.; Dubosclard-Gottardi, C. Synth. Commun. 1996, 26, 2811.

(c) Mouradzadegun, A.; Ganjali, M. R.; Mostafavi, M. A. Appl. Organomet. Chem. 2018, 32, e4214.

(d) Cao, Y. Q.; Chen, B. H.; Pei, B. G. Synth. Commun. 2001, 31, 2203.

[4] (a) Ren, Y.; Yan, M.; Zhao, S. Tetrahedron Lett. 2011, 52, 5107.

(b) Saha, D.; Adak, L.; Mukherjee, M. Org. Biomol. Chem. 2012, 10,952

(c) Xia, A.; Xie, X.; Chen, H. Org. Lett. 2018, 20, 773.

(d) Ratani, T. S.; Bachman, S.; Fu, G. C. J. Am. Chem. Soc. 2015, 137, 13902 .

(e) Nenajdenko, V. G.; Muzalevskiy, V. M.; Shastin, A. V. J. Fluorine Chem. 2007, 128, 818 .

(f) Wang C, Wang C, Wang Q. Chem.-Eur. J. 2007, 13, 6484.

[5] (a) Yabe, O.; Mizufune, H.; Ikemoto, T. Synlett 2009, 1291.

(b) Satoh, Y.; Obora, Y. RSC Adv. 2014, 4, 15736.

(c) Zieger, H. E.; Wo, S. J. Org. Chem. 1994, 59, 3838.

(d) Echigo, Y.; Watanabe, Y.; Mukaiyama, T. Chem. Lett. 1977, 6, 697. (e) Munemori, D.; Tsuji, H.; Uchida, K. Synthesis 2014, 46, 2747.

[6] Powell, K. J.; Han, L. C.; Sharma, P. Org. Lett. 2014, 16, 2158.

[7] Dowd, P.; Wilk, B. K.; Wlostowski, M. Synth. Commun. 1993, 23, 2323.

[8] Shapiro, E. A.; Pereverzeva, Y. O.; Nefedov, A. O.; Éismont, M. Y. Russ. Chem. B 1989, 38, 2432.

[9] (a) Meng, H.; Gao, S.; Luo, M. Eur. J. Org. Chem. 2006, 4617.

(b) Geng, H.; Huang, P. Q. Tetrahedron 2015, 71, 3795.

(c) Lammens, T. M.; Le Nôtre, J.; Franssen, M. C. ChemSusChem. 2011, 4, 785 .

(d) Dai, J. J.; Huang, Y. B.; Fang, C. ChemSusChem 2012, 5, 617. (e) Cook, M. C.; Witherell, R. D.; White, R. L. Lett. Drug Des. Discovery 2010, 7, 9 .

(f) Temelli, B.; Unaleroglu, C. Synthesis 2014, 46, 1407. (g) Barhdadi, R.; Gal, J.; Heintz, M. Tetrahedron 1993, 49, 5091. (h) Black, P. J.; Edwards, M. G.; Williams, J. M. Eur. J. Org. Chem. 2006, 4367.

(i) Camps, F.; Gasol, V.; Guerrero, A. Synth. Commun. 1988, 18, 445. (j) Boivin, J; Laurent, K. E.; Zard, S. Z. Tetrahedron 1995, 51, 2573.

(k) Ghiaci, M.; Sedaghat, M. E.; Kalbasi, R. J. Tetrahedron 2005, 61, 5529.

(1) DiBiase, S. A.; Wolak, R. P.; Dishong, D. M. J. Org. Chem. 1980, 45, 3630 .

(m) Guaragna, A.; Mauro, D. N.; Pedatella, P. J. Labelled Compd. Radiopharm. 2006, 49, 675.

(n) Hu, L. Z; Hussain, M. I.; Deng, Q. F.; Liu, Q.; Feng, Y. Y; Zhang, X. H.; Xiong, Y. Tetrahedron 2019, 75, 308.

(o) Laroche, C.; Harakat, D.; Bertus, P. Org. Biomol. Chem. 2005, 3,3482 .

(p) Jouanno, L. A.; Sabot, C.; Renard, P. Y. J. Org. Chem. 2012. 77,8549 .

(q) Christophe, L.; Vincent, C.; Ludwig, C.; Cyril, O.; Louis, F. Eur. J. Org. Chem. 2017. 2118.

(Lu, Y.) 